\title{
Micron-sized atom traps made from magneto-optical thin films
}

\author{
S. Eriksson, ${ }^{1}$ F. Ramirez-Martinez,${ }^{1}$ E.A. Curtis,${ }^{1}$ B.E. Sauer,${ }^{1}$ P.W. Nutter,${ }^{2}$ E.W. Hill, ${ }^{2}$ and E.A. Hinds ${ }^{1}$ \\ ${ }^{1}$ Blackett Laboratory, Imperial College, London $S W^{7} 2 B W$, United Kingdom \\ ${ }^{2}$ Department of Computer Science, University of Manchester, Manchester M13 9PL, United Kingdom
}

\begin{abstract}
We have produced magnetic patterns suitable for trapping and manipulating neutral atoms on a $1 \mu \mathrm{m}$ length scale. The required patterns are made in $\mathrm{Co} / \mathrm{Pt}$ thin films on a silicon substrate, using the heat from a focussed laser beam to induce controlled domain reversal. In this way we draw lines and "paint" shaped areas of reversed magnetization with sub-micron resolution. These structures produce magnetic microtraps above the surface that are suitable for holding rubidium atoms with trap frequencies as high as $\sim 1 \mathrm{MHz}$.
\end{abstract}

PACS numbers: 39.25.+k, 03.75.Be, 75.50.Ss, 75.70.-i

\section{INTRODUCTION}

The study of cold atom clouds in microscopic magnetic traps and waveguides has recently become a vigorous field of research [1, 2]. In particular, the ability to confine and manipulate atoms above microstructured surfaces known as atom chips - holds great promise for integrated atom optics and the realisation of new quantum devices. Examples include miniature atom interferometers 3, 4] and quantum information processors [5].

Most atom chip experiments to date have produced the trapping fields by passing current through integrated wires. This approach has two undesirable features. First, magnetic field noise is generated by thermal fluctuation of the charges in the wires [6, 7]. This can change the internal state of the atoms through magnetic dipole spinflip transitions [8, 9], causing decoherence and trap loss. Second, the current does not flow as intended within the wires, but wanders from side to side 10, resulting in traps of uneven depth. An atom cloud cooled in such a trap breaks into fragments when the available energy becomes comparable with the amplitude of the noise in the trap depth 10, 11, 12]. These imperfections are detrimental for many practical applications.

As an alternative, our group has been working on microscopic patterns of permanent magnetisation [1], which offer a way around the problems discussed above by avoiding currents and by keeping the metal layers thin $\mathbf{7}$, 8]. Previous experiments used videotape [8, 13], on which patterns with spatial periods as small as $10 \mu \mathrm{m}$ are conveniently written. Future experiments will aim for controlled tunnelling of atoms between one trap and another as a powerful way to prepare quantum states of the atomic motion [14. This will require magnetic structures of order $1 \mu \mathrm{m}$ in size, prompting us to investigate $\mathrm{Co} / \mathrm{Pt}$ thin films. Small structures also make traps so strong that atom clouds can be compressed down to one dimension for new experiments in the physics of quantum gases [15, 16].

In this paper, we describe the fabrication of atomic microtraps based on magneto-optically (MO) patterned $\mathrm{Co} / \mathrm{Pt}$ thin films. When combined with a suitable uniform external field, the magnetic field above the pat- terned surface of the film creates local minima where alkali atoms prepared in a weak-field-seeking state can be confined [1]. The first attempt to do this was by the group of P. Hannaford using TbFeCo films, but with these it did not seem possible to achieve the desired control over the domain boundaries [17]. Here we show by contrast that $\mathrm{Co} / \mathrm{Pt}$ films are suitable for writing well-defined structures on the required $1 \mu \mathrm{m}$ size scale. The strong magnetisation of the cobalt in conjunction with the small scale of the structures produces large magnetic field gradients of order $10^{4} \mathrm{~T} / \mathrm{m}$. The magnetic field noise will be low because the metal film is thin. This will allow trapped rubidium atoms to be held at micron distances from the surface where the strong field gradient gives trapping frequencies in excess of $1 \mathrm{MHz}$, corresponding to exceedingly tight atom confinement.

The magnetisation of the film is normal to the surface, providing the freedom to create arbitrary $2 \mathrm{D}$ patterns in the plane of the film. This is a key difference between in-plane and perpendicular magnetic media. The writing technique used in this work is based on a standard method used in data storage applications and has a similar aim, i.e. to produce small scale patterns. However, high density data storage aims to achieve small isolated spots on the film, whereas our aim is to produce larger, uniformly magnetised regions with high constrast and small feature sizes along the boundary. MO techniques have not previously been used for this purpose. Here we present the writing of two patterns that are fundamental building blocks for atom trapping and manipulation. The writing process is fully reversible: any pattern can be erased and re-written, and in principle this could even be done in the presence of trapped atoms. This offers an advantage in comparison with lithographically prepared atom chips, which cannot be reconfigured.

In the next section we describe the preparation of $\mathrm{Co} / \mathrm{Pt}$ multilayer thin films and we summarise their magnetic properties. Section [II] describes the experimental apparatus and the procedure for writing. SectionIV is devoted to analysis of the patterns that were written and a discussion of their application to atom chips. We conclude with a summary of our results. 


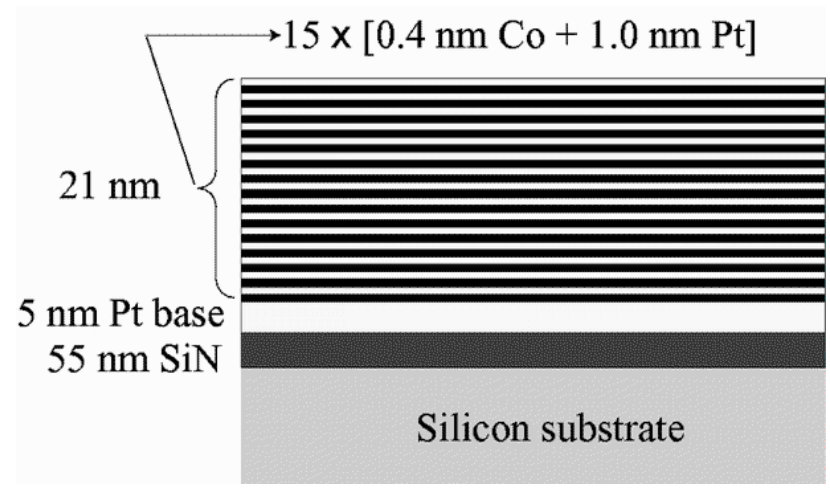

FIG. 1: Layer structure used for the Co/Pt thin films (not to scale).

\section{THE Co/Pt MULTILAYER THIN FILMS}

Magneto-optical films have been much studied for their application to data-storage [18]. Recently, Co/Pt films such as we are using here have attracted interest because they give a strong optical Kerr rotation to blue light, which can have a small spot size. High resolution makes $\mathrm{Co} / \mathrm{Pt}$ attractive for writing microscopic atom traps and guides as well as for data storage. Other relevant properties are a strong saturation magnetisation and a large perpendicular anisotropy, leading to a large coercivity and a very square hysteresis loop [19]. The perpendicular anisotropy depends strongly on the crystallographic orientation of the layers: a thick Pt base layer increases the coercivity dramatically by establishing good $\{111\}$ texture on which to deposit the multilayer. The squareness of the hysteresis loop is optimised by having control over the layer thicknesses: $0.2-0.4 \mathrm{~nm}$ for the Co, corresponding to 1-2 monolayers, and $\sim 1 \mathrm{~nm}$ for the $\mathrm{Pt}$ layers 20].

The specific layer structure that we use is shown in Fig.11 First, the silicon substrate has a $55 \mathrm{~nm}$-thick silicon nitride layer, which seems necessary to achieve MO recording. In our first attempts on silicon we did not have this insulating layer and we found that the $180 \mathrm{~mW}$ of laser power available for writing was not enough to make any observable change in the film, either physical or magnetic. This problem is rectified by the silicon nitride layer, which lowers the laser power required for writing, presumably because it increases the thermal resistance between the magnetic layers that must be heated and the silicon wafer that acts as a heat sink.

The Pt and Co layers are laid down by vacuum deposition using an electron beam source to evaporate the metals. The base pressure before evaporation is $2 \times 10^{-7}$ Torr, and the average pressure during deposition rises to $3-4 \times 10^{-7}$ Torr. We heat the substrate to a temperature of $200 \mathrm{C}$ in order to establish adequate $\{111\}$ texture with a platinum base layer that is only $5 \mathrm{~nm}$ thick 19$]$. A thicker layer gives better texture, but inhibits the MO writing by allowing the heat to flow too quickly away

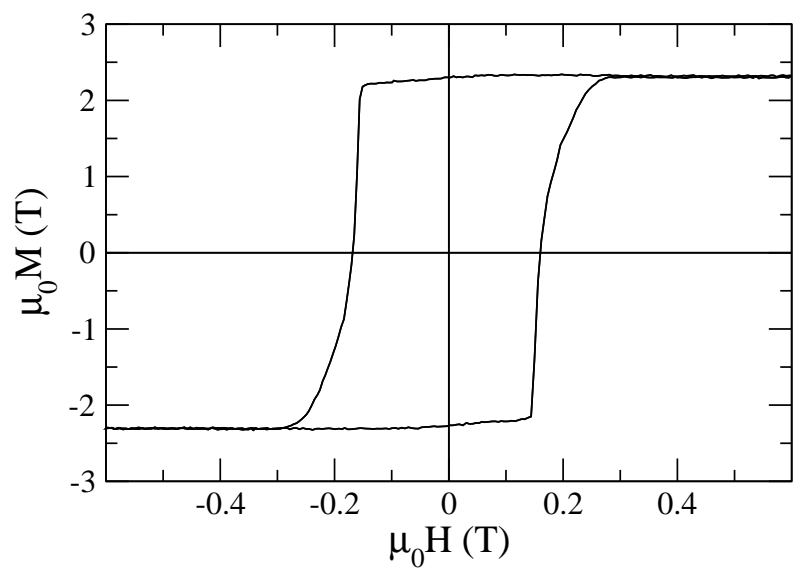

FIG. 2: Alternating gradient force magnetometer measurement of the hysteresis curve. Here the magnetic moment of the film is expressed as an effective magnetisation within the $6 \mathrm{~nm}$ thickness of the Co layers.

from the laser spot. The magneto-optical film itself consists of 15 bi-layers of alternating $\mathrm{Co}(0.4 \mathrm{~nm})$ and $\mathrm{Pt}$ $(1.0 \mathrm{~nm})$. The evaporation rates are kept low at $10 \mathrm{pm} / \mathrm{s}$ to ensure a good control of the layer thicknesses.

The magnetic properties were determined using an alternating gradient force magnetometer, which yielded the hysteresis curve shown in Fig.2] The measured coercivity is given by $\mu_{0} H_{c}=0.16 \mathrm{~T}$. The measured magnetic moment can be converted to an effective average magnetisation within the net $6 \mathrm{~nm}$ thickness of the cobalt. Expressed in this way, the magnetisation at saturation is given by $\mu_{0} M_{s}=2.3 \mathrm{~T}$, some $30 \%$ higher than that of bulk cobalt. This enhancement can be attributed to additional magnetisation coming from polarized Pt atoms near the Co layers [19].

\section{WRITING ON THE FILMS}

The writing procedure depends upon the reduction of the coercivity with increasing temperature [21]. The film is initially driven into saturation in one direction normal to the surface by a strong external magnetic field. This field is then reduced below the room temperature coercivity and reversed. By illuminating the sample locally with a focused laser beam, very small areas of the film can be selectively heated and with an appropriate movement of either the film or the laser beam, patterns of reversed magnetization are created.

\section{A. Apparatus}

After using a permanent magnet to saturate it, the film is placed between the pole faces of an electromagnet as shown schematically in Fig. 3 This provides a reverse field of order $100 \mathrm{mT}$ for the purpose of writing patterns 


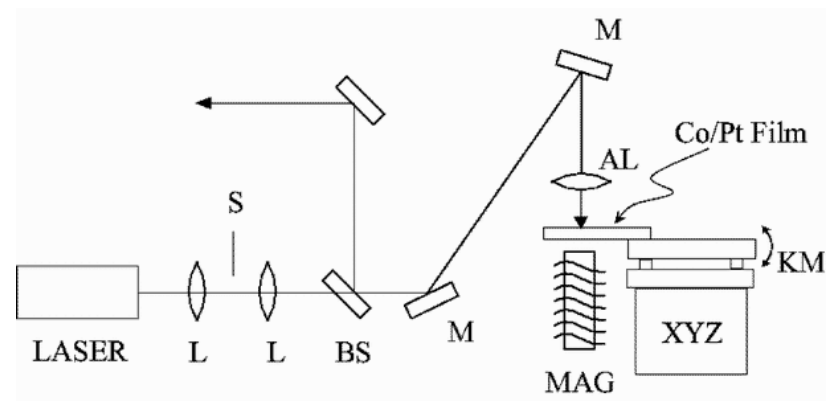

FIG. 3: Experimental setup for thermomagnetic writing. Abbreviations: (L) lenses, (S) programmable shutter, (BS) beam-splitter, (M) mirrors, (AL) aspheric lens, (MAG) electromagnet, (KM) kinematic mount, and (XYZ) translation stages.

on the film. The laser used for writing is a frequency doubled Nd:YVO laser operating at $532 \mathrm{~nm}$ on the $\mathrm{TM}_{00}$ transverse mode. The first lens $\mathrm{L}$ focuses the light to a waist, where a mechanical shutter $\mathrm{S}$ is used to switch the beam on and off. A second identical lens recollimates it. Two mirrors $\mathrm{M}$ steer the beam to an aspheric lens $\mathrm{AL}$ with 0.55 numerical aperture and $4.4 \mathrm{~mm}$ focal length that focuses the light to a (calculated) waist of $0.6 \mu \mathrm{m}$, i.e. this is the radius at which the intensity drops to $1 / e^{2}$ of its peak value. The $\mathrm{Co} / \mathrm{Pt}$ film sits horizontally in a tray attached to a three point kinematic mount KM, which is connected in turn to an $x y z$ translation stage.

Approximately $54 \%$ of the light is reflected, allowing us to position the film in the focal plane by translating it along the vertical $z$ direction until the retro-reflected beam is collimated. A beam-splitter BS picks off the back reflection for this purpose. Since the Rayleigh length of the focussed light is only $2 \mu \mathrm{m}$, this requires delicate adjustment, for which we use a differential micrometer.

In order to write magnetic patterns, the film is moved horizontally by a translation stage under computer control. Initially, the plane of this $x y$ translation does not coincide exactly with the surface of the film, but through iterative adjustments of the kinematic mount, we make the two planes coincide so that the spot is always focussed on the surface. The next two subsections describe how we use this setup to write patterns suitable for atom trapping.

\section{B. Writing an array of lines}

Our first pattern is a set of parallel lines, as shown in Fig.4] With the reversed magnetic field set at $50 \mathrm{mT}$ and the laser power at $75 \mathrm{~mW}$, the sample is translated at $250 \mu \mathrm{m} / \mathrm{s}$ along the $x$ axis. This produces a line of reverse magnetisation $1 \mu \mathrm{m}$ wide, which can be as long as the size of the film. At the end of each line, the shutter is closed and the sample is moved back to the start before making a step of $2 \mu \mathrm{m}$ along $y$ to the start of the next line.

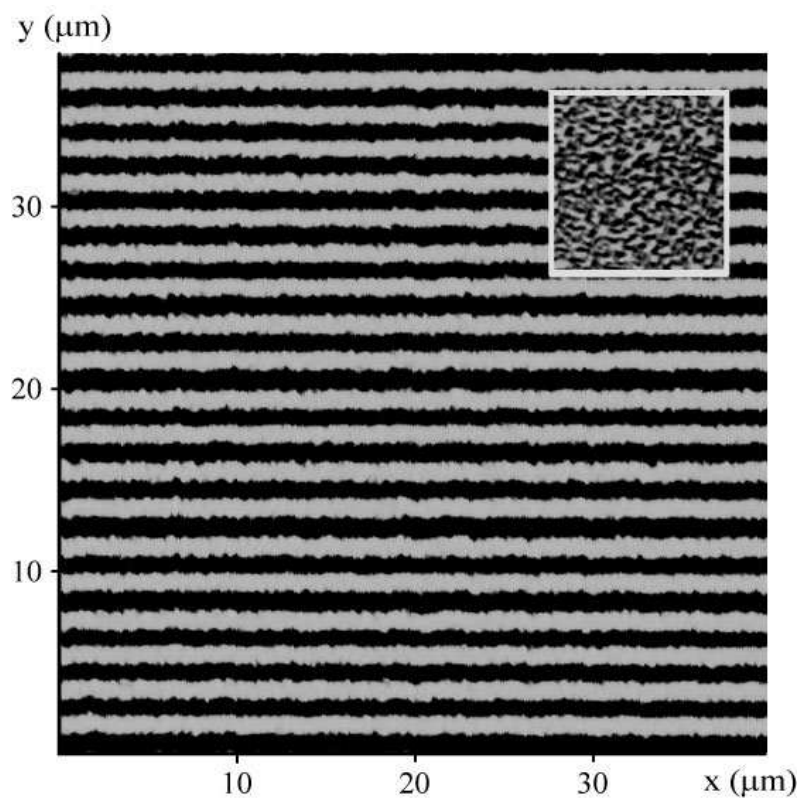

FIG. 4: Magnetic force microscope image showing part of a pattern of parallel lines written on the film. The dark regions are unchanged from the original magnetisation, whereas the bright regions show where the magnetisation is reversed. Inset: a nearly demagnetised piece of the $\mathrm{Co} / \mathrm{Pt}$ film, showing the size of the domains.

The particular array shown in Fig. 4 covered a $200 \mu \mathrm{m}$ square area. The figure shows a typical small section of the array imaged by a magnetic force microscope. The dark regions indicate areas magnetised in the original direction whilst the bright regions have reverse magnetisation. Such images give quantitative information about the positions and widths of the lines. They also allow us to see when a region is magnetically saturated. If the film is demagnetised, the domains are mixed, with some magnetised up and others down as shown inset in the top right hand corner of Fig. [4 By contrast, the uniformity of the lines in Fig 4 shows that their magnetization is saturated. Along the boundaries between lines, one can see small irregularities: the lines waver by approximately $250 \mathrm{~nm}$ peak-to-peak. The period of this noise is typically $300 \mathrm{~nm}$ but the spectral distribution is broad. We have not been able to achieve straighter boundaries and we presume that lines written in this way have a fundamental noise level imposed by the domain size.

This method of writing only works for a relatively narrow range of parameters. For example, if the laser power is lowered to $70 \mathrm{~mW}$, there is hardly any magnetic response to the writing procedure. However, if the scanning speed is reduced at the same time, the film responds once again. This seems reasonable as it must surely be the temperature reached in the magnetic film that is the essential parameter. If instead we increase the laser power to $80 \mathrm{~mW}$, we start to see physical damage to the surface of the film. Atomic force microscope measurements show a $2 \mathrm{~nm}$ high ridge along the line drawn by the laser at this 


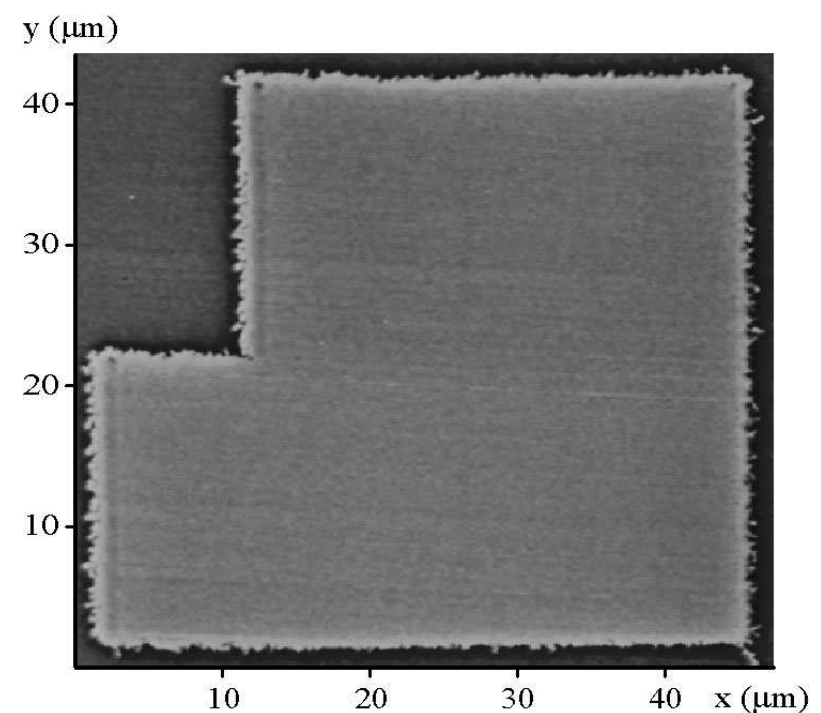

FIG. 5: Magnetic force microscope scan of a Z-trap pattern. The grey-scale in this image is identical to that in Fig.4

power. This can be avoided by making a compensating change to higher scan rate. There are also constraints on the strength of the reverse magnetic field bias. Below $50 \mathrm{mT}$, we start to see domain structure in the body of the lines indicating that the remagnetisation is not saturated. Above $100 \mathrm{mT}$ the domain irregularities along the boundary lines begin to grow. For this reason, we operate at the lowest reverse field that still saturates the body of the lines.

\section{Painting an area}

Our second pattern involves "painting" an area of reversed magnetisation in the shape of a $40 \mu \mathrm{m}$ square with a piece missing from the top left, as shown in Fig.5] The motivation for making this particular shape is discussed in the next section. Once again the pattern is made by drawing a series of lines at $250 \mu \mathrm{m} / \mathrm{s}$ along the $x$ direction, but in this case the step along $y$ is $0.5 \mu \mathrm{m}$, which makes successive lines overlap. In order to achieve full overlap of the lines we we used a higher laser power of $79 \mathrm{~mW}$ and increased the reverse bias field to $0.1 \mathrm{~T}$.

The edges parallel to the $x$ axis have a similar level of noise to that seen in Fig.4. However, the transverse deviations can be as large as $1 \mu \mathrm{m}$ peak-to-peak on the edges parallel to $y$. Here the translation stage has decelerated as it approaches the end of the line, resulting in an excess deposition of energy. In combination with the larger reverse bias, this promotes domain growth, leading to the protuberances seen in the picture. We anticipate that this can be remedied by more precise control over the shutter timing, which would allow us to start and end the lines while the stage is still moving at constant velocity. Further, improved actuation along the $y$ direc- tion would allow us to use a shorter step size, so that the pattern could be written with a lower bias field and less laser power. We believe that the noise along the edges of the pattern can be reduced at least to the same level as seen for the array of lines with these modifications of the apparatus.

\section{APPLICATION TO ATOM MICROTRAPS}

In thinking about the kinds of fields that could be made above the surface, it is useful to replace the magnetisation by an equivalent current density $\nabla \times M[22]$. Along a boundary where the magnetisation reverses, going from $M$ to $-M$, this current density results in an equivalent current of $2 M t$, where $t$ is the thickness of the magnetised material. Taking our value of $2.3 \mathrm{~T} / \mu_{0}$ for the cobalt magnetisation and $6 \mathrm{~nm}$ for the thickness of the cobalt, we obtain an equivalent current of $22 \mathrm{~mA}$ along boundaries where the magnetisation reverses. Thus, the structures we can make in this magnetic film are equivalent to any network of $22 \mathrm{~mA}$ current loops in a plane.

Viewed in this way, the pattern shown in Fig.4 is equivalent to lines of current parallel to the $x$ axis, spaced by $1 \mu \mathrm{m}$ and alternating in direction. The magnetic field they produce is easily calculated using the Biot-Savart law. A similar pattern with $3 \mu \mathrm{m}$ period was recently fabricated by etching a hard disk drive 23]. Larger structures of this kind have previously been fabricated on videotape and used as atom mirrors [1] and gratings [13]. Here, however, we are primarily interested in the application to trapping because of the unusually high trap frequencies that can be reached in small-scale structures. To be specific, let us consider adding a uniform bias field of $2.6 \mathrm{mT}$ along the $y$ direction to interfere with the field created by the film. Fig.6(a) shows the resulting contours of constant field strength. The circles in the contour plot enclose lines of zero magnetic field that are formed by destructive interference at a height $z$ of $0.75 \mu \mathrm{m}$ in a $2 \mu \mathrm{m}$-period array. Weak-field-seeking atoms may be trapped on these lines. An atom held in one of these traps will lose its spin orientation if the field is allowed to go strictly to zero, so we include here a small uniform field $B_{0}=0.1 \mathrm{mT}$ along the $x$ direction to maintain the quantization.

Figs.6(b) and (c) show the field strength versus $y$ and $z$. In both cases this varies linearly in the vicinity of the trap with a gradient of $B^{\prime}=8.5 \times 10^{3} \mathrm{~T} / \mathrm{m}$, except in the central $10-20 \mathrm{~nm}$, where the bias $B_{0}$ is significant. There the field strength grows quadratically, leading to a transverse oscillation frequency for the trapped atom given approximately by

$$
f=\frac{1}{2 \pi} \sqrt{\frac{\mu}{m} \frac{B^{\prime 2}}{B_{0}}},
$$

where $\mu$ and $m$ are the magnetic moment and mass of the atom. For ${ }^{87} \mathrm{Rb}$ atoms in the $F=2, m_{F}=2$ state, the 

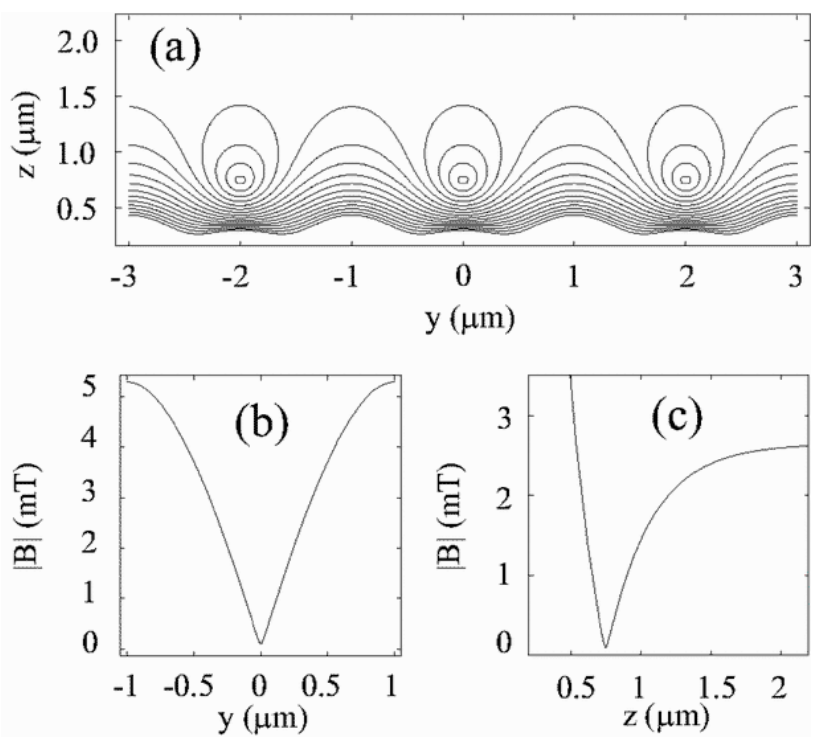

FIG. 6: Calculated magnetic field magnitude above an array of lines (see Fig. (4) with a $2.6 \mathrm{mT}$ bias along $y$ to form the traps and $0.1 \mathrm{mT}$ along $x$ to preserve the atomic spin orientation. (a) Contours of constant field strength. (b) Field strength versus $y$ through the centre of a trap at $z=0.75 \mu \mathrm{m}$. (c) Field strength versus $z$ through the centre of a trap at $y=0$.

trap in this example has a very high oscillation frequency of $f=1.1 \mathrm{MHz}$.

We turn now to the $x$ direction, along the axis of the trap. There is no trapping force in this direction, but axial confinement can be achieved if necessary by additional current-carrying wires underneath the chip. For example, two wires positioned at $x= \pm 1000 \mu \mathrm{m}, z=-750 \mu \mathrm{m}$, each carrying $10 \mathrm{~A}$ along $y$, will confine rubidium atoms in the axial direction with an oscillation frequency of $82 \mathrm{~Hz}$. This is the type of arrangement normally used in our group [10].

A more accurate description of the field above the film must take into account the domain structure seen in Fig.4 Thus, the equivalent currents do not really flow along the $x$ direction but oscillate from side to side in the $x y$ plane. The typical amplitude is $a=125 \mathrm{~nm}$ and the typical wavelength is $\lambda=300 \mathrm{~nm}$. At height $z>>a$ above such a wire, the main field component is

$$
B_{y} \simeq \mu_{0} I / 2 \pi z
$$

whilst the transverse oscillations produce an oscillating field along the wire given by [10]

$$
B_{x} \simeq \frac{\mu_{0} I}{2 \pi} k^{2} a K_{1}(k z) \cos (k x),
$$

where $k=2 \pi / \lambda$ and $K_{1}$ is the modified Bessel function. It is this latter field component that makes the traps uneven and causes atom clouds to break up above currentcarrying wires. In the present case of Rb atoms $750 \mathrm{~nm}$ above the film, this field has an amplitude of $\sim 10 \mathrm{nT}$,
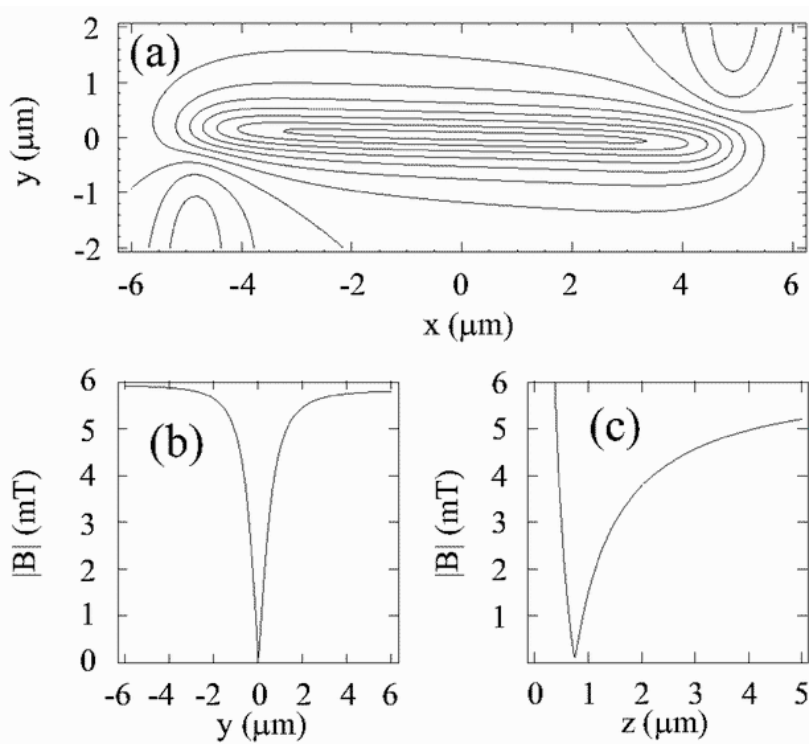

FIG. 7: Calculated magnetic field strength near the Z-trap of Fig. 5 with $5.8 \mathrm{mT}$ bias along y to form the trap. (a) Contours of constant field strength. (b) Field strength versus $y$ through centre of trap at $z=0.75 \mu \mathrm{m}$. (c) Field strength through trap centre versus $z$.

corresponding to an undulating potential of $\sim 10 \mathrm{nK}$. For many applications this amount of roughness is perfectly acceptable, but for the most sensitive experiments on quantum gases and quantum information processing it is not. We believe that further improvement would be worthwhile and could be achieved by having smaller domain sizes. Alternative writing procedures are also under investigation.

For the magnetic pattern shown in Fig. [5] the equivalent current is a single loop encircling the region of reversed magnetisation. The Z-shaped edge on the left is the region of interest for atom trapping purposes. The short central section along $x$ is the "wire" above which atoms are to be trapped, whilst the longer legs serve as the end wires, providing both axial confinement 24] and a suitable bias of $0.12 \mathrm{mT}$ along $x$ for avoiding spin flip transitions. For a $22 \mathrm{~mA}$ current flowing in this way, we calculate that a bias field of $5.8 \mathrm{mT}$ along $y$ makes a trap lying $0.75 \mu \mathrm{m}$ above the film. Fig. 7(a) shows the contours of constant magnetic field strength in the $x y$ plane $0.75 \mu \mathrm{m}$ above the film, with the origin defined to be at the centre of the trap. Curves (b) and (c) show cuts through the trap in the $y$ and $z$ directions. The transverse oscillation frequency along $y$ or $z$ is $0.9 \mathrm{MHz}$. In the axial direction along $x$ it is $19 \mathrm{kHz}$ and in this case no auxiliary wires are needed. If a more anisotropic trap is required, one simply has to make the central section of the Z-shaped boundary be longer. 


\section{SUMMARY}

We have shown that areas and lines of saturated magnetisation can be written thermomagnetically on $\mathrm{Co} / \mathrm{Pt}$ thin films. We have illustrated the method with two patterns that are relevant for producing high frequency neutral atom traps: the long wire and the Z-trap. With these drawing and painting tools any pattern of interest can be made at a resolution down to approximately $1 \mu \mathrm{m}$. This small scale gives access to extremely tight traps with $\mathrm{MHz}$ frequencies for rubidium atoms, making the method promising for studies of $1 \mathrm{D}$ quantum gases and for small atom trap arrays that could be suitable for quantum information processing.

In atom chips based on current-carrying wires, the magnetic field close to a wire suffers from rf interference and thermal noise, which cause atom loss through vibrational excitations and spin flips. These effects should be avoided with the use of the $\mathrm{Co} / \mathrm{Pt}$ thin films. A further problem with current-carrying wires is that the current wanders from side to side, leading to uneven traps. A similar effect is found in the $\mathrm{Co} / \mathrm{Pt}$ thin films due to the domain structure. However the amplitude and period of these excursions are small compared with a micron, making the effect negligible in all but the tightest traps, i.e. those that are closest to the surface.

\section{Acknowledgments}

We are indebted to Jon Dyne for expert technical assistance. We also thank David Lau and Boris Vodungbo for their contributions to the early stages of the project. This work was supported by the UK Engineering and Physical Sciences Research Council and by the FASTNET network of the European Union.
[1] E.A. Hinds and I.A. Hughes, J. Phys. D: Appl. Phys. 32, R119 (1999)

[2] R. Folman, P. Krüger, J. Schmiedmayer, J. Denschlag, and C. Henkel, Adv. At. Mol. Opt. Phys. 48, 263 (2002).

[3] E.A. Hinds, C.J. Vale, and M.G. Boshier, Phys. Rev. Lett. 86, 1462 (2001).

[4] W. Hänsel, J. Reichel, P. Hommelhoff, and T.W. Hänsch, Phys. Rev. A 64, 063607 (2001).

[5] T. Calarco, E.A. Hinds, D. Jaksch, J. Schmiedmayer, J.I. Cirac, and P. Zoller, Phys. Rev. A 61, 022304 (2000).

[6] C. Henkel, S. Pötting, and M. Wilkens, Appl. Phys. B 69, 379 (1999); C. Henkel, and M. Wilkens, Europhys. Lett. 47, 414 (1999).

[7] P.K. Rekdal, S. Scheel, P.L. Knight, and E.A. Hinds, quant-ph/0403166 (2004); Phys. Rev. A. in press (2004).

[8] M.P.A. Jones, C.J. Vale, D. Sahagun, B.V. Hall, and E.A. Hinds, Phys. Rev. Lett. 91, 080401 (2003).

[9] D.M. Harber, J.M. McGuirk, J.M. Obrecht, and E.A. Cornell, J. Low. Temp. Phys. 133, 229 (2003).

[10] M.P.A. Jones, C.J. Vale, D. Sahagun, B.V. Hall, C.C. Eberlein, B.E. Sauer, K. Furusawa, D. Richardson, and E.A. Hinds, J. Phys B 37, L15 (2004).

[11] J. Fortágh, H. Ott, S. Kraft, A. Günter, and C. Zimmermann, Phys. Rev. A 66, 041604(R) (2002).

[12] A.E. Leanhardt, Y. Shin, A.P. Chikkatur, D. Kielpinski, W. Ketterle, and D. Pritchard, Phys. Rev. Lett. 90, 100404 (2003).

[13] P. Rosenbusch, B.V. Hall, I.G. Hughes, C.V. Saba, and E.A. Hinds, App. Phys. B 70, 709 (2000).

[14] M. Greiner, O. Mandel, T. Esslinger, T.W. Hänsch, and
I. Bloch, Nature 415, 39 (2002).

[15] U. Al Khawaja, J.O. Andersen, N.P. Proukakis, and H.T.C Stoof: Phys. Rev. A. 66, 013615 (2002).

[16] V. Dunjko, V. Lorent, and M. Olshanii, Phys. Rev. Lett. 86, 5413 (2001).

[17] D.C. Lau, R.J. McLean, A.I. Sidorov, D.S. Gough, J. Koperski, W.J. Rowlands, B.A. Sexton, G.I. Opat, and P. Hannaford, J. Opt. B: Quantum Semiclass. Opt, 1, 371 (1999).

[18] Mansuripur: Principles of magneto-optical recording (Cambridge University Press, 1995).

[19] C.J. Lin, G.L. Gorman, C.H. Lee, R.F.C. Farrow, E.E. Marinero, H.V. Do, H. Notarys, and C.J. Lin, J. Magn. Magn. Mater. 93, 194 (1991).

[20] W.B. Zeper, F.J.A.M. Greidanus, and P.F. Carcia, IEEE Trans Magn. 25, 3764 (1989); W.B. Zeper, H.W. van Kesteren, B.A.J. Jacobs, and J.H.M. Spruit, J. Appl. Phys. 70, 2264 (1991).

[21] W.W. Clegg, N.A.E. Heyes, E.W. Hill, and C.D. Wright, J. Magn. Magn. Mater. 95, 49 (1991); C.D. Wright, W.W. Clegg, A. Boudjemline, and N.A.E. Heyes, Jap. J. Appl. Phys, 33, 2058 (1994).

[22] J.D. Jackson: Classical Electrodynamics 3rd Edn. (Wiley, NewYork,1998).

[23] B. Lev, Y. Lassailly, C. Lee, A. Scherer, and H. Mabuchi, Appl. Phys. Lett. 83, 395 (2003).

[24] J. Reichel, W. Hänsel, and T.W. Hänsch, Phys. Rev. Lett. 83, (1999), 3398. 